\title{
Response to the Letter by Dr. Farzad Gheshlaghi
}

\section{Sudheera Jayasinghe}

Published online: 13 March 2012

(C) American College of Medical Toxicology 2012

\section{Dear Editor,}

We would like to respond to the letter by Dr. Farzad Gheshlaghi on the article entitled "Effects of Deliberate Ingestion of Organophosphate or Paraquat on Brain Stem Auditory-Evoked Potentials (BAEP)"

The study group was interested in finding out whether there is any clinically significant lesion in the auditory pathway following acute ingestion of organophosphate (OP) or paraquat (PQ). History and examination of all the patients confirmed that they had acute ingestion of OP or PQ.

For our OP patients, quantification of plasma cholinesterase (ChE) activity within $12 \mathrm{~h}$ of ingestion was available in $34 / 70$. There was no statistically significant correlation of ChE activity with $1-111,111-\mathrm{V}$, and $1-\mathrm{V}$ latencies (Table 1).

PQ is extremely toxic with a $65 \%$ fatality following suicidal ingestion. We assessed BAEP of PQ survivors. Since PQ is rapidly absorbed from gastrointestinal tract and has high affinity for tissue, early sample collection to determine plasma PQ levels is required to estimate severity of ingestion. We were unable to do this, but all the patients in the study had positive urine paraquat to sodium dithionite test.

Nevertheless, further studies with the assessment of auditory threshold and serial BAEP in acute OP or PQ poisoning are needed.

We thank Dr. Gheshlaghi for his interest and comments.

Table 1 Correlation of ChE activity with 1-111, 111-V, and 1-V latencies of BAEP

\begin{tabular}{lrr}
\hline Latency & Right side & Left side \\
\hline $1-111$ & $0.2(0.3)$ & $0.1(0.5)$ \\
$111-\mathrm{V}$ & $0.3(0.1)$ & $0.1(0.5)$ \\
$1-\mathrm{V}$ & $-0.02(0.9)$ & $0.2(0.5)$ \\
\hline
\end{tabular}

Values are Spearman's correlation coefficient ( $P$ value) 